\title{
Validity of the Relative Percent Concept for Equating Training Intensity
}

\author{
Victor Katch, Arthur Weltman ${ }^{1}$, Stanley Sady, and Patty Freedson \\ Physical Performance Research Laboratory, \\ Physical Education Department, The University of Michigan, \\ 401 Washtenaw Avenue, Ann Arbor, MI 48109, U.S.A.
}

\begin{abstract}
Summary. The validity of the relative percent concept for equating training intensity was investigated using the point of metabolic acidosis (anaerobic threshold) as the criterion variable. Percent oxygen uptake, heart rate and metabolic acidosis were determined at 60,70 , and $80 \%$ of max heart rate on a bicycle ergometer test $(N=31)$. At 60 and $70 \%$ of max heart rate only one individual was definitely in metabolic acidosis. At $80 \%$ max heart rate 17 subjects were working at a level above the point of metabolic acidosis while 14 were working at or below this point. Thus, it was suggested that even if subjects are exercising at the same relative percent maximum HR, themetabolic stress using metabolic acidosis as the criterion is not constant across subjects. It was further shown that the regression of percent $\mathrm{VO}_{2}$ max on percent max HR was a spurious one resulting in poor prediction of individual $\mathrm{VO}_{2}$ values. The data presented suggest that the relative percent concept for equating exercise or subsequent training intensity needs careful re-evaluation.
\end{abstract}

Key words: Training $-\dot{V} \mathrm{O}_{2} \max -$ Anaerobic threshold - Metabolic acidosis - Intensity - Exercise.

There is considerable controversy concerning various physical training protocol variables and their relative contribution to the training effect. Existing evidence seems to indicate that intensity of training is the key independent variable which determines changes in the dependent variables chosen to represent the training effect (Burke, 1975; Crews, 1976; Davies, 1971; Faria, 1975; Nupp, 1970; Pollock, 1973; Sharkey, 1970). Other variables may also be important, with some investigators clearly pointing out the considerable interaction effect between intensity, mode, frequency, and duration (Crews, 1976; Haslam, 1974; Hellerstein, 1973; Sharkey, 1970; Wenger, 1975).

1 Present address: Department of Physical Education, University of Louisville, Louisville, KY, U.S.A.

Offprint requests to: V. Katch (address see above) 
With few exceptions, most physical training studies are designed in such a way that one or several of the above variables are assigned to subjects based on what may be termed the "relative percent concept". This procedure usually involves a pretraining graded exercise stress test with the measurement of peak or maximum physiological values (e.g., max heart rate or maximum oxygen uptake). On the basis of these test results a relative percent of the peak values are assigned as the training stimulus. The most common procedure is to use percent maximum heart rate (\% $\max (H R)$ as the criterion reference point. If a subject attains a peak HR of 210 beats per min, for example, and using $80 \%$ as the training stimulus, the corresponding assigned training exercise would be 168 beats per min. Using this procedure it is possible to have all subjects train at the same relative percent with the assumption that the work intensity has been 'equated' between subjects. There are numerous examples of this procedure in the literature (Davies, 1971; Durnin, 1960; Fox, 1973, 1975; Jackson, 1968; Nupp, 1970; Sharkey, 1970).

The converse to this relative percent technique would be to use absolute work or physiological scores as the training stimulus. That is, have everyone train at the same absolute heart rate e.g., $160 \mathrm{bpm}$, etc. This method appears to be less desirable, however, since most investigators apparently feel that if persons of different fitness levels train at the same absolute heart rate then the stress to each individual would be markedly different and would not allow for a fair comparison of the training effect.

The purpose of this paper is to discuss the validity of the relative percent concept with special reference to use of percent max $\mathrm{HR}$ as an indicator of percent $\dot{V} \mathrm{O}_{2}$ max (\% $\dot{V}_{2} \max$ ).

\section{Special Considerations}

The basis behind use of the relative percent concept is the regression of percent $\dot{V O} \mathrm{O}_{2}$ max on percent max HR. Since there are individual differences in the slope of individual regression lines (Skinner, 1974), use of percent values makes it possible to use a common regression equation for all subjects. From this regression line, which is assumed to be essentially linear, estimates of percent $\dot{V O}_{2}$ max are made from percent max HR.

An example of a scatter plot of percent $\mathrm{VO}_{2}$ max on percent max HR is shown in Figure 1. These data are based on percent $\dot{V} \mathrm{O}_{2}$ and percent $H R$ values at the different work loads for $N=31$ male subjects who completed a progressive resistance bicycle ergometer stress test. The test was started at zero-load and every $3 \mathrm{~min}$ thereafter the resistance was increased $0.5 \mathrm{kp}(180 \mathrm{kpm})$. The RPM was established at 60 . The test was terminated when the subjects would no longer turn the pedals. Standard open circuit spirometric techniques were used to obtain min-by-min oxygen uptake (Wilmore, 1968 ). $\dot{V} \mathrm{O}_{2}$ max was designated as the peak $\dot{V} \mathrm{O}_{2}$ uptake in the series of consecutive scores for each individual. In all cases this was reached within the last 2 min of exercise. Min-by-min heart rate was recorded using a standard hard-wire EKG unit. Work-output was recorded by a microswitch mounted on the frame of the bike which was activated by each pedal revolution. 


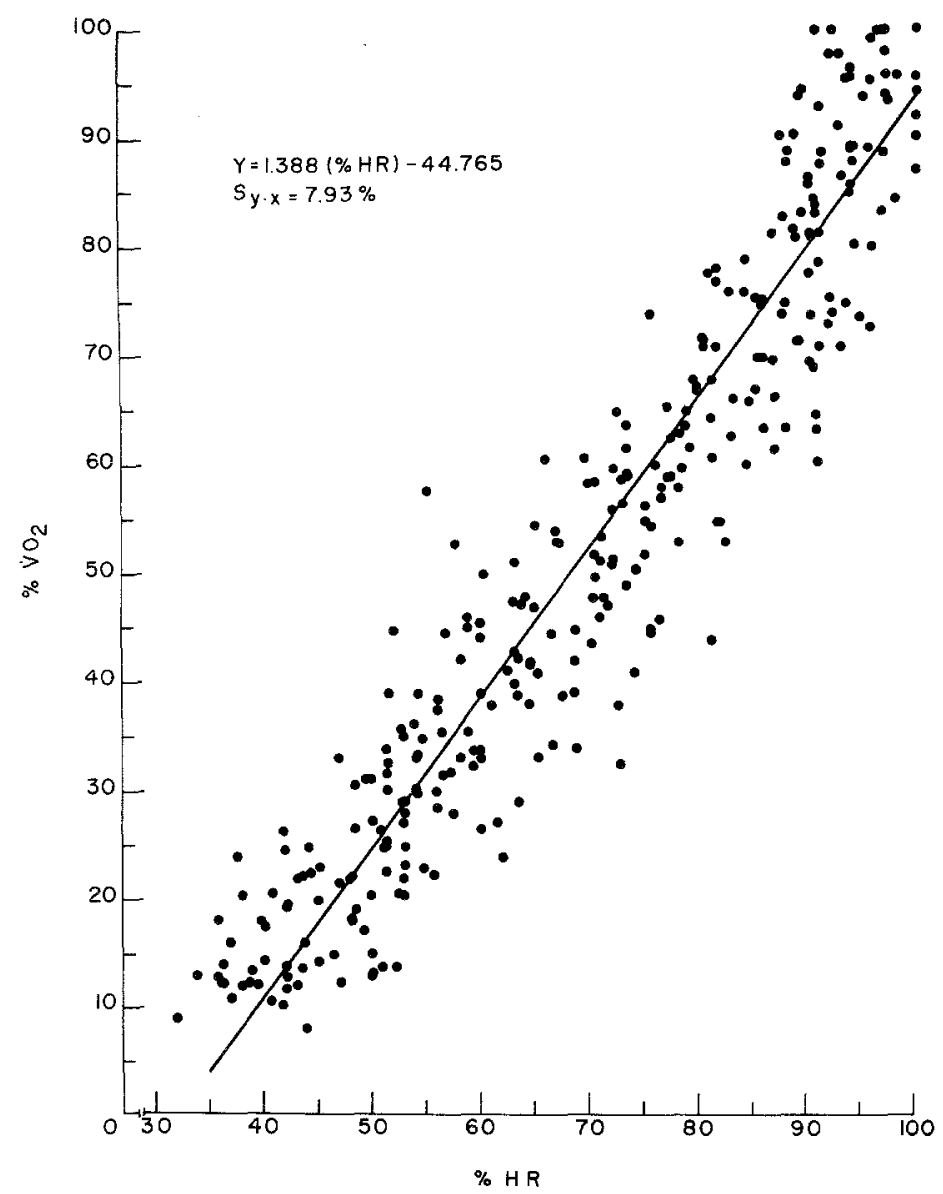

Fig. 1. Scatter plot and best fit straight line between percent max. HR versus percent $\dot{V} \mathrm{O}_{2} \max$ for $N=31$ male subjects

The individual percent $\mathrm{VO}_{2}$ and $\mathrm{HR}$ data points were fit with a linear regression equation of the form $Y^{\prime}=m X+C$, where $Y^{\prime}=$ predicted percent $\dot{V O}_{2} \max$, $m=$ slope of the line, $\left(r_{x, y}\right)\left(\mathrm{SD}_{Y}\right) /\left(\mathrm{SD}_{x}\right)$, and $C=$ additive constant $(Y$ intercept). While it is possible to fit these data with higher order polynominals, as done by Londeree and Ames (1976), the increase in the accountability of unexplained variance is of no practical significance. Our listed slope and intercept are nearly indentical to that reported by others (Londeree, 1976; Saltin, 1968; Skinner, 1974; Taylor, 1969).

The correlation between percent $\dot{V} \mathrm{O}_{2}$ max and percent max HR is very high in the present example $(r=0.97)$. Nevertheless, it is essential to point out that this relationship is a spurious one owing to the fact that both variables are work-rate and time dependent. That is, assume that the correlation between $\dot{V O}_{2}$ and HR were zero, but within a given individual as work-rate increases so does both $\mathrm{HR}$ and $\dot{V} \mathrm{O}_{2}$. This generates a correlation since the respective bivariate scores ( $\mathrm{HR}$ and $\dot{V}_{2}$ ) become 
ranked as work increases. If one plots the HR and $\mathrm{VO}_{2}$ scores for a group of individuals a substantial correlation has been manufactured, when in fact the correlation is really zero. Since the rate of performing work on the increment test is time dependent (i.e., higher work outputs necessarily result in longer riding times), and $\dot{V} \mathrm{O}_{2}$ uptake and $\mathrm{HR}$ are proportional to work output up to maximum within individuals, it follows that $\dot{V O}_{2}$ uptake and HR must also be time dependent on this type of increment test. Accordingly, the within test correlation between percent $\dot{V O}_{2} \max$ and percent max. HR contains the common element of time. When removing this common element from the two variables by the partial correlation technique, the $r=0.97$ drops to $r=0.17$.

Using the partial correlation in the above example is perhaps not a completely fair test of the extent the correlation is spurious since the partial $r$ is holding constant any variability in the work output. However, it does illustrate that by making use of simultaneous measures of $\mathrm{HR}, \dot{V} \mathrm{O}_{2}$, and work output, the correlation between HR and $\mathrm{VO}_{2}$ will necessarily be high whether or not a true relationship exists.

The high correlation between percent $\mathrm{VO}_{2}$ max and percent max HR implies a high correlation between the actual $\dot{V O}_{2} \max$ and max HR scores. In the present data the correlation between $\mathrm{VO}_{2} \max$ and $\max \mathrm{HR}$ is $r=-0.03$. Also, the correlations between $\dot{V} \mathrm{O}_{2}$ and HR during any given minute of the test does not exceed $r=0.50$ ( $25 \%$ common variance). This is similar to data reported elsewhere (Damato, 1966; Girandola, 1971). Thus, attempting to predict levels of energy expenditure (\% $\mathrm{VO}_{2}$ max) from sub-maximum HR using mean regression analyses will result in gross miscalculations of individual values. Girandola et al. (1971) reached the same experimental conclusions using completely different analyses.

\section{Validity of the Relative Percent Concept}

Attempts to validate the relative percent $\mathrm{HR}$ procedure for assigning training intensity is complicated by choice of appropriate criterion scores. In general, $\mathrm{VO}_{2}$ max is often used as the criterion score. However, this procedure is also complicated as there is evidence indicating that the $\mathrm{VO}_{2}$ max is itself a poor predictor of physiological functioning. Factors such as differences in body size (i.e., lean body weight to fat composition ratio), aging effects and heredity, differentially affect this measure. In addition, the $\dot{V}_{2}$ max may not discriminate adequately between subjects for variables that may change as a result of physical training.

Recent evidence presented by Wasserman et al. (1973) suggests that measurement of the onset of metabolic acidosis (termed the anaerobic threshold, AT) allows a more complete description of the circulatory and metabolic adaptations to graded exercise stress than is afforded by only measuring the $\dot{V O}_{2}$ max. It has long been known that if an external workload is increased in a linear fashion from light to heavy the oxygen uptake response will follow linearly. The energy supply for the low level work, where the oxygen demand is equal to oxygen supply, are derived primarily oxidatively. On the other hand in heavy exercise the oxygen demand exceeds the oxygen supply requiring the tapping of anaerobic reserves, presumably via glycolytic mechanisms. Thus, during incremental exercise a workload and energy level exists where there is an imbalance of the oxygen demand-supply relationship resulting in 
increased lactic acid formation (Wasserman, 1973). The measurement of this workload or energy level at which the onset of anaerobiosis occurs offers information regarding the circulatory, metabolic and energy source-supply adaptations not obtained by measuring the $\mathrm{VO}_{2}$ max. In fact, many investigators set up training protocols at specific heart rates under the notion that the corresponding $\dot{V} \mathrm{O}_{2}$ 's that subjects are exercising at are above or below the point where lactic acid is being produced.

The work of Holloszy (1973) suggests that the effects of physical training are probably more evident in subtle internal cellular adaptations which may or may not be manifested in gross changes in the $\mathrm{VO}_{2}$ max. In this respect, the AT can be viewed as a reasonable criterion variable which to validate the relative percent HR procedure. Theoretically, training at intensities below or above this level should result in different physiological changes. That is, there is reason to believe that if an individual trains at an intensity which is below the level of anaerobiosis the primary fuel substrate for exercise will be fat [for a complete discussion see Holloszy (1973)]; thus, there should be greater changes in body composition than if one were to train at levels above the point of anaerobiosis. Conversely, training at intensity levels above the AT should result in greater changes in cardio-respiratory parameters than when training below the AT.

The feasibility of measuring the AT with the description of a non-invasive procedure was reported by Naimark et al. (1964) and more recently by Wasserman et al. (1973). The AT is defined as the workload or $\mathrm{VO}_{2}$ just below the point of non-linear increases in the respiratory exchange ratio, $(R)$ minute ventilation $\left(\dot{V}_{E \mathrm{BTPS}}\right)$ and $\dot{V} \mathrm{CO}_{2}$ production during incremental exercise. The validity of this procedure has recently been reported by Davis et al. (1976) who found a correlation of $r=0.95$ between AT measured via gas exchange measures $\left(\dot{V}_{E}, \dot{V} \mathrm{CO}_{2}, F_{e} \mathrm{O}_{2}\right.$, and $\left.F_{e} \mathrm{CO}_{2}\right)$ and AT measured via continuous measurements of venous lactate. Additionally, no significant differences $(p>0.05)$ where observed between the means for the two methods for measuring the AT.

From a validity standpoint the question arises as to the relationship between the physiological parameters recorded at various percents of $\max \mathrm{HR}$, as for example $60,70,80$, and an individual's AT. Stated simply, if individuals train at $80 \% \max$ HR is the response in relation to the AT the same between subjects? If it is not, this would suggest that even though individuals are working at the same relative percent of max HR, the related stress and expected training changes will be different between subjects. This would indicate that the use of the relative percent concept is invalid, at least in terms of using percent $\mathrm{HR}$ as the training stimulus.

Table 1 shows the physical characteristics, $\mathrm{VO}_{2}$ max, and AT for the 31 subjects whose data appear in Figure 1. Procedures outlined by Wasserman et al. (1973) and Davis et al. (1976) were used to record the AT. Ventilation expired (BTPS) $\dot{V} \mathrm{CO}_{2}$, $R, F_{E} \mathrm{O}_{2}$ and $F_{E} \mathrm{CO}_{2}$ were plotted versus work rate and time using the third minute value at each work level. The AT was designated for each test as the workload just below the point of departure from linearity in the $\dot{V}_{E}$ and $\hat{V C O}_{2}$ responses, together with abrupt increases in $R$ and $F_{E} \mathrm{O}_{2}$. The AT was independently determined by two investigators using any combination of the criterion variables. Of the 31 plots we examined only three discrepancies existed. These three discrepancies were arbitrated by a third investigator. Recording the exact workload and time where the AT is 
Table 1. Physical characteristics and related variables of subjects, $N=31$

\begin{tabular}{lrrl}
\hline Variable & Mean & SD & Range \\
\hline Age, years & 22.65 & 3.42 & $18-31$ \\
Weight, $\mathrm{kg}$ & 72.86 & 10.03 & $57.8-98.2$ \\
$\mathrm{Height}, \mathrm{cm}$ & 177.41 & 6.90 & $164.6-187.4$ \\
$\dot{V} \mathrm{O}_{2} \max , 1 \cdot \mathrm{min}^{-1}$ & 3.74 & 0.65 & $2.53-5.77$ \\
$\dot{\mathrm{VO}} \mathrm{max}, \mathrm{ml} \cdot \mathrm{kg}^{-1} \cdot \mathrm{min}^{-1}$ & 51.36 & 6.36 & $37.8-68.8$ \\
$\mathrm{Max} . \mathrm{HR}, \mathrm{bpm}$ & 197.40 & 7.60 & $180-216$ \\
Anaerobic threshold, $\mathrm{kpm}$ & 972.72 & 250.51 & $513-1416$ \\
$\dot{V} \mathrm{O}_{2}$ uptake at anaerobic threshold, & 2.23 & 0.52 & $1.27-3.36$ \\
$1 \cdot \min ^{-1}\left(\mathrm{VO}_{2}-\mathrm{AT}\right)$ & & & \\
\end{tabular}

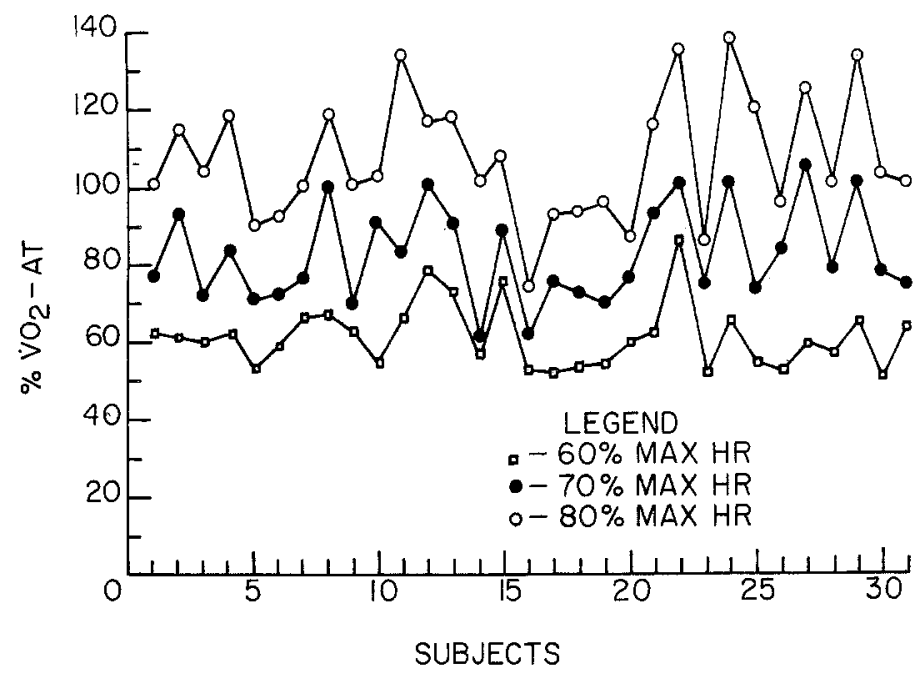

Fig. 2. Plot of individual subject's percent of anaerobic threshold (AT) at the different percent max. HR. On the right axis is labeled the corresponding oxygen uptake for the respective percent AT

reached and reading off the corresponding $\dot{V} \mathrm{O}_{2}$ uptake makes it possible to express the AT in oxygen uptake units. This value is termed the $\dot{V} \mathrm{O}_{2}-\mathrm{AT}$.

For each subject the associated variables corresponding to 60,70 , and $80 \%$ of max HR were recorded. For example, for a subject whose max HR is $200 \mathrm{bpm}$ his $60 \% \mathrm{HR}$ would be $120 \mathrm{bpm}$. At the $120 \mathrm{HR}$ his associated $\dot{\mathrm{VO}_{2}}$ uptake would be $1.251 \cdot \mathrm{min}^{-1}$, which corresponds to $60 \%$ of his $\dot{V} \mathrm{O}_{2}$-AT and $35 \%$ of his measured $\mathrm{VO}_{2}$ max. This procedure was followed for each subject for 60,70 , and $80 \% \max$ HR. These percentages were used as they represent the most common percent HR training levels.

The mean data for the above variables are given in Table 2. The percent $\dot{V} \mathrm{O}_{2}$ max values corresponding to the 60,70 , and $80 \% \max \mathrm{HR}$ are similar to values reported in the literature (Pollock, 1973). What is important to emphasize is that if subjects were training at 60 or $70 \%$ max HR they essentially would be training at a 
Table 2. Related criterion variables determined at 60,70 , and $80 \%$ of maximum heart rate. See text for full explanation

\begin{tabular}{lccc}
\hline Variable & \multicolumn{3}{l}{ Percent max. HR condition } \\
\cline { 2 - 4 } & $60 \%$ & $70 \%$ & $80 \%$ \\
\hline Oxygen uptake, $1 \cdot \min ^{-1}$ & $1.36 \pm 0.35$ & $1.82 \pm 0.47$ & $2.36 \pm 0.55$ \\
Percent $\dot{V} \mathrm{O}_{2}$ max & $36.4 \pm 6.6$ & $48.4 \pm 8.2$ & $62.5 \pm 7.9$ \\
Percent $\dot{V} \mathrm{O}_{2}-\mathrm{AT}$ & $61.2 \pm 8.4$ & $81.8 \pm 12.3$ & $106.6 \pm 15.9$ \\
Number of subjects above $\dot{V O}_{2}-\mathrm{AT}$ & 0 & 1 & 17 \\
Number of subjects at or below $\dot{V} \mathrm{O}_{2}-\mathrm{AT}$ & 31 & 30 & 14 \\
\hline
\end{tabular}

a Values listed are mean $\pm \mathrm{SD}$

level below the AT. Consequently, one would not expect significant changes to occur in cardio-respiratory functioning. Results from several training studies where subjects trained at these percentages of maximum HR supports this contention (Davies, 1971; Fox, 1973; Sharkey, 1970).

At $80 \%$ max. HR, which corresponds to $62 \%$ of $\dot{V} \mathrm{O}_{2} \max ( \pm 7.9 \%$ ), 17 of the subjects would be training at or above the AT level. Thus, one would expect greater cardio-respiratory changes than in the other two \% HR conditions. However, it should also be noted that 14 subjects would be training at a level below the AT. These data are presented graphically in Figure 2.

An additional comment is warranted here. It is common practice during treadmill tests to maintain a constant speed and to alter the percent grade every 3 min or so until volitional exhaustion. The corresponding $\mathrm{VO}_{2}$ and $\mathrm{HR}$ are recorded at predetermined time intervals in order to establish an individual's percent $\mathrm{HR}-\dot{\mathrm{VO}} \mathrm{O}_{2}$ curve. Individuals are then required to train at a given percent HR on a level track. In order to attain this percent HR individuals must run at speeds which are different than those used during the stress test in the laboratory. No data to our knowledge are available showing that the same slope of the $\mathrm{HR}-\dot{V} \mathrm{O}_{2}$ line exists during constant speed-variable grade and constant grade-variable speed conditions. It is highly probable, however, in light of data showing different efficiencies and oxygen requirements (Gaesser, 1975) as a function of speed of movement that there will be differing $\mathrm{HR}-\dot{\mathrm{V}} \mathrm{O}_{2}$ relationships.

\section{Additional Comments}

It seems that indiscriminate use of the relative percent concept, as typically used to equate physical training stimuli may result in erroneous experimental conclusions.

In a practical sense if the relative percent concept, as applied to HR during training may not be stressing individuals equally in terms of energy mobilization, what is the alternative? Perhaps a better method for equating the training stimulus between subjects might be to equate individuals based on their anaerobic threshold. In terms of expected changes and stress on the energy systems the AT seems to be a 
well-chosen criterion. The procedures for measuring the AT are similar in design to a standard stress-test and, in fact, can be incorporated in such a test. But, as pointed out by Wasserman et al. (1973) the HR is a poor indicator of the AT (owing to large intra-subject variability); therefore, it would be necessary to use some other variable, as for example work rate as the equating variable.

As the test protocol for determining the AT has only been reported for the bicycle ergometer further research is needed to extend this analysis to the treadmill. If it should turn out that a dependable relationship exists between $\dot{V} \mathrm{O}_{2}$-AT and running speed (at $0 \%$ grade), then running speed could be used as the equating variable.

In summary, caution and reservation is advised in selecting, using, and interpreting data based on training intensities which rely on the relative percent of max HR as the training stimulus. Further studies and critical evaluations are necessary.

\section{References}

Burke, E. J., Franks, D. B.: Changes in $\mathrm{VO}_{2}$ max resulting from bicycle training at different intensities holding total mechanical work constant. Res. Quart. Amer. Ass. Hlth. Phys. Educ. 46, 31-37 (1975)

Crews, T. R., Roberts, J. A.: Effects of interaction of frequency and intensity of training. Res. Quart. Amer. Ass. Hith. Phys. Educ. 47, 48-55 (1976)

Damato, A. N.: Hemodynamic response to treadmill exercise in normal subjects. J. Appl. Physiol. 21, 959-966 (1966)

Davis, J. A., Vodak, P., Wilmore, J. H., Vodak, J., Kurtz, P.: Anaerobic threshold and maximal oxygen uptake for three modes of exercise. J. Appl. Physiol. 41, 544-550 (1976)

Davies, C. T. M., Knibbs, A. V.: The training stimulus: The effects of intensity, duration and frequency on max aerobic power output. Int. Z. Angew. Physiol. 29, 299-304 (1971)

Durnin, J. V. G.: Effects of a short period of training of varying severity in some measurements of physical fitness. J. Appl. Physiol. 15, 161-165 (1960)

Faria, I. E.: Cardiovascular response to exercise as influenced by training of various intensities. Res. Quart. Amer. Ass. Hlth. Phys. Educ. 46, 31-37 (1975)

Fox, E. L., Mathews, T. K.: Intensity and distance of interval training programs and changes in aerobic power. Med. Sci. Sports 5, 18-22 (1973)

Fox, E. L., Mathews, T. K.: Frequency and duration of interval training programs and changes in aerobic power. J. Appl. Physiol. 38, 481-484 (1975)

Gaesser, G., Brooks, G.: Muscular efficiency during steady-state exercise: Effects of speed and work rate. J. Appl. Physiol. 38, 1132-1139 (1975)

Girandola, R. N., Katch, F. I., Henry, F. M.: Prediction of oxygen intake from ventilation, and oxygen intake and work capacity from heart rate during heavy exercise. Res. Quart. Amer. Ass. Hith. Phys. Educ. 42, 362-373 (1971)

Haslam, R. W., Stull, G. A.: Duration and frequency of training as determinants of coronary tree capacity in the rat. Res. Quart. Amer. Ass. Hlth. Phys. Educ. 45, 178--184 (1974)

Hellerstein, H. K., Hirsch, E. L., Ader, R., Greenblot, N., Siegel, M.: Principles of exercise prescription. In: Exercise testing and exercise training in coronary heart disease (J. P. Naughton, H. K. Hellerstein, eds.), pp. 129-169. New York: Academic Press 1973

Holloszy, J.: Biochemical adaptations to exercise: Aerobic metabolism. In: Exercise and sport science reviews (J. H. Wilmore, ed.). New York: Academic Press 1973

Jackson, J. H.: Cardiorespiratory adaptations to training at specified frequencies. Res. Quart. Amer. Ass. Hlth. Phys. Educ. 39, 295-300 (1968)

Londeree, B. R., Ames, S. A.: Trend analysis of $\% \dot{V O}_{2}$ max-Hr regressions. Med. Sci. Sports 8, $122-125(1976)$ 
Naimark, A., Wasserman, K., Mcllroy, M. B.: Continuous measurement of ventilatory exchange ratio during exercise. J. Appl. Physiol. 19, 644-652 (1964)

Nupp, W. F.: The relative effectiveness of four cardio-respiratory conditioning programs. I. Sport Med. (Torino) 10, 84-89 (1970)

Pollock, M. L.: The quantification of endurance training programs. In: Exercise and sport science reviews (J. A. Wilmore, ed.). New York: Academic Press 1973

Saltin, B., Blonquist, B., Mitchell, J. H., Johnson, R. L., Jr., Wildenthal, K., Chapman, C. B.: Response to submaximal and maximal exercise after bed rest and training. Circulation 38 (Suppl. 7), 19-20, $40-41,63-69$ (1968)

Sharkey, B. J.: Intensity and duration of training and the development of cardio-respiratory endurance. Med. Sci. Sports 2, 197-202 (1970)

Skinner, J. S., Jankowski, L. W.: Individual variability in the relationship between heart rate and oxygen intake. Med. Sci. Sports 6, 68 (1974)

Taylor, H. L., Haskell, W., Fox III, S. M., Blackburn, H.: Exercise tests: A summary of procedures and concepts of stress testing of cardiovascular diagnosis and function evaluation. In: Measurement in electrocardiography (H. Blackburn, ed.), pp. 259-305. Springfield: Thomas 1969

Wasserman, K., Whipp, B., Koyal, S., Beaver, W.: Anaerobic threshold and respiratory gas exchange during exercise. J. Appl. Physiol. 35, 236-243 (1973)

Wenger, J. A., MacNab, R. B. J.: Endurance training: The effects of intensity, duration and initial fitness. J. Sport Med. (Torino) 15, 199-211 (1975)

Wilmore, J. H.: A simplified method for obtaining aliquots of respiratory air. Res. Quart. Amer. Ass. Hith. Phys. Educ. 39, 324-328 (1968)

Accepted May 5, 1978 